\title{
EQUIVARIANT HOMOLOGY DECOMPOSITIONS
}

\author{
PETER J. KAHN
}

\begin{abstract}
This paper presents some results on the existence of homology decompositions in the context of the equivariant homotopy theory of Bredon. To avoid certain obstructions to the existence of equivariant Moore spaces occurring already in classical equivariant homotopy theory, most of the work of this paper is done "over the rationals." The standard construction of homology decompositions by Eckmann and Hilton can be followed in the present equivariant context until it is necessary to produce appropriate $k^{\prime}$-invariants. For these, the Eckmann-Hilton construction uses a certain Universal Coefficient Theorem for homotopy sets. The relevant extension of this to the equivariant situation is an equivariant Federer spectral sequence, which is developed in \$2. Using this, we can formulate conditions which imply the existence of the desired $k^{\prime}$-invariants, and hence the existence of the homology decomposition. The conditions involve a certain notion of projective dimension. For one application, equivariant homology decompositions always exist when the group has prime order.
\end{abstract}

1. Introduction and statement of results. This note presents some results on the existence of homology decompositions in the context of equivariant homotopy theory. $G$ will always denote a finite group, and $G$-spaces and $G$-maps will always be pointed, unless explicitly stated otherwise.

By equivariant homotopy theory, we mean the framework developed originally by Bredon [B]. Thus, the reduced homology $\underline{\tilde{H}}_{*} X$ of a $G$-space $X$ is a certain gradedabelian-group-valued functor that incorporates all the homology information of the $H$-fixed sets $X^{H}$ as $H$ ranges over the subgroups of $G$. Analogous formulations apply to other standard functors of algebraic topology, e.g., to homotopy groups $\underline{\pi}_{n} X$. (See 2.1 or $[\mathbf{B}]$ for more details.)

A homology decomposition of a $G$-space $X$ is defined when each $X^{H}$ is 1connected, i.e., when $\underline{\pi}_{n}(X)=0$, for $n \leq 1$. It consists of a filtered $G$-space

$$
K=\lim _{\longrightarrow} K_{m} \supset \cdots \supset K_{m} \supset \cdots \supset K_{2} \supset K_{1}=*
$$

and a $G$-map $e: K \rightarrow X$ which is a homology equivalence, such that (a) $\underline{H}_{i} K_{m}=$ $\underline{H}_{i} K, i \leq m$; (b) $\underline{\tilde{H}}_{i}\left(K_{m}\right)=0, i>m$; (c) each $K_{m+1}$ is obtained from $K_{m}$ by equivariantly attaching the cone $\hat{c} A_{m+1}$ on a certain $G$-space $A_{m+1}$. More precisely, the $G$-space $A_{m+1}$ satisfies (i) $\underline{\pi}_{i} A_{m+1}=0, i<m$; (ii) $\underline{\tilde{H}}_{i} A_{m+1}=0, i>m$; (iii) the functor $\underline{\tilde{H}}_{m} A_{m+1}$ is naturally equivalent to $\underline{\tilde{H}}_{m+1} X$. When $m \geq 2$, we summarize (i)-(iii) by saying that $A_{m+1}$ is a Moore $G$-space of type $\left(\underline{\tilde{H}}_{m+1} X, m\right)$, or that $A_{m+1}$ realizes $\underline{\tilde{H}}_{m+1} X$ in degree $m$.

Received by the editors April 22, 1985.

1980 Mathematics Subject Classification (1985 Revision). Primary 55P99, 57S17, 55P62.

Key words and phrases. Equivariant, homology decomposition, Moore space.

The author was partly supported by NSF Grant DMS-8201029. 
When $G=\{e\}, e$ the identity element, the preceding definition reduces to the standard, nonequivariant one. When fixed sets $X^{H}$ are ignored for all $H \neq$ $e$, then the preceding defines what we call a "classical" (equivariant) homology decomposition and classical Moore $G$-space. The functors $\underline{\tilde{H}}_{m} A_{m+1}$ and $\underline{\tilde{H}}_{m+1} X$, etc. then reduce to ordinary homology groups viewed as (left) modules over the group ring $\mathbf{Z} G$.

Unfortunately, even this simpler classical equivariant homology decomposition of $X$ may fail to exist for an obvious reason. Namely, it may be that some of the Z $G$-modules $\tilde{H}_{m+1} X$ cannot be realized in degree $m$ by a classical Moore $G$-space. For an example of this, first choose $G$ and a $\mathbf{Z} G$-module $M$ that is not realizable in some degree $m \geq 2$. Such $G$ and $M$ are described in $[\mathbf{C}, \mathbf{K 1}, \mathbf{S m}]$. Then let $X$ be a classical Eilenberg-Mac Lane $G$-space of type $(M, m+1)$. These exist for all $M$ and all $m \geq 0$ (e.g., see [Ro]).

We wish to avoid such obvious obstacles. That is, we wish to be in a situation in which all the needed Moore $G$-spaces do exist. We then attempt to determine what, if any, further conditions are needed for obtaining a homology decomposition.

These considerations motivate the following conditions, which we shall impose on most of our results.

(1) Abelian groups are vector spaces over $\mathbf{Q}$ (the rational numbers).

(2) Spaces are pointed and 1-connected and have finite-type over $\mathbf{Q}$.

Note that if $X$ is a $G$-space, then (1) and (2) taken together require that each $X^{H}$ be pointed and 1-connected and have rational homotopy and homology groups which are finite-dimensional $\mathbf{Q}$-vector spaces.

Conditions ( $\mathbf{Q})$ do have the desired effect. That is, assuming (Q), a theorem of Triantafillou [T1] applies to show that Moore $G$-spaces of all types exist (cf. [K2]).

Our general existence theorem now requires one further condition, because of the complexity of the homological algebra of the functors $\underline{\tilde{H}}_{i} X$. In particular, the projective dimension $\underline{\tilde{H}}_{i} X$ may be large, whereas, by way of contrast, all $\mathbf{Q} G$ modules are projective. To describe the condition, we need the following notation:

$$
\begin{aligned}
\underline{\operatorname{conn}} X & =\sup \left\{i \mid \underline{\pi}_{j}(X)=0, j=0,1, \ldots, i\right\}, \\
\gamma(m, X) & =\inf \left\{i \mid i \geq 1 \text { and } \underline{\tilde{H}}_{m+i}(X) \neq 0\right\} .
\end{aligned}
$$

We shall say that the homology of $X$ "has gaps" if $\gamma(m, X) \geq 2$ for all $m$.

THEOREM A. Suppose $X$ is a $G$-space satisfying $(\mathbf{Q})$ and, for each $m$,

$$
\text { proj } \operatorname{dim} \underline{\tilde{H}}_{m} X \leq \min \{\underline{\operatorname{conn}} X, \gamma(m, X)\} .
$$

Then $X$ admits a homology decomposition.

This has the following immediate consequence which avoids reference to conn.

COROllary B. Suppose that $X$ is a finite-dimensional $G$-space satisfying $(\mathbf{Q})$ and, for each $m$,

$$
\text { proj } \operatorname{dim} \underline{\tilde{H}}_{m} X \leq \gamma(m, X) \text {. }
$$

Then some suspension of $X$ has a homology decomposition.

The next corollary feeds in some known facts about projective dimension to produce specific examples. 
COROLlary C. Let $X$ be a G-space satisfying (Q). In each of the following cases, $X$ has a homology decomposition.

(a) $G$ is a cyclic p-group.

(b) $G=\mathbf{Z} / p \oplus \mathbf{Z} / p$ and the homology of $X$ has gaps.

(c) $X$ is an Eilenberg-Mac Lane $G$-space of type $(\underline{\pi}, n)$ with $n>\log _{2}|G|$.

A better lower bound for $n$ in (c) is described in the proof of Corollary C in $\S 4$.

THEOREM D. Let $X$ be a $G$-space satisfying

(a) conn $X \geq 1$;

(b) proj $\operatorname{dim} \underline{\tilde{H}}_{m} X \leq 1$, for all $m$.

Then $X$ admits a homology decomposition.

Note that this theorem does not assume $(\mathbf{Q})$. When $(\mathbf{Q})$ is assumed, then, of course, $D$ is an immediate consequence of Theorem $\mathrm{A}$.

Our next result shows when we can obtain classical homology decompositions.

COROLlaRY E. Suppose that $X$ is a $G$-space satisfying

(a) $X$ is 1-connected;

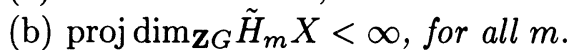

Then $X$ admits a classical (equivariant) homology decomposition.

Note that (a) and (b) of this corollary are "classical" analogues of corresponding hypotheses in Theorem D. Typical cases in which (b) is satisfied occur when $X$ satisfies $(\mathbf{Q})$ or is $p$-local or $p$-complete for some prime $p$ not dividing $|G|$.

Next we give an example showing that the conditions in Theorem A and Corollary $\mathrm{B}$ are sharp.

THEOREM F. Let $G=\mathbf{Z} / 2 \oplus \mathbf{Z} / 2$. There exists a 5 -dimensional $G$-complex $X$ satisfying $(\mathbf{Q})$ and

(a) $\underline{\operatorname{conn}} X=2$;

(b) $\gamma(3, X)=1$;

(c) proj $\operatorname{dim} \underline{\tilde{H}}_{3} X=2$;

(d) neither $X$ nor any suspension of $X$ admits a homology decomposition.

The notion of (nonequivariant) homology decomposition is due originally to $\mathrm{J}$. Moore $[\mathbf{M}]$ and to B. Eckmann and P. Hilton (see $[\mathbf{H}]$ ). Their procedure for constructing such decompositions (cf. [H, p. $87 \mathrm{ff}]$ ) carries over in a straightforward manner to the equivariant context, except for the argument that produces the $k^{\prime}$ invariant. In the nonequivariant case, this is produced via a short-exact "universal coefficient sequence" for homotopy with coefficients $[\mathbf{H}]$. In the equivariant case such a short-exact sequence must be replaced by a spectral sequence, and the conditions we impose in, say, Theorem $\mathrm{A}$ are used to conclude that a certain portion of the spectral sequence collapses, again producing the desired $k^{\prime}$-invariant.

The spectral sequence in question is, essentially, an equivariant Federer spectral sequence. While it is clearly related to the spectral sequence of $[\mathbf{S c}]$, the viewpoints of the two results appear to be quite different, and there does not seem to be a brief translation from one to the other. To describe the spectral sequence, we use the following notation. Recall that all $G$-spaces and $G$-maps are pointed. Given $G$-spaces $X, Y$, let $[X, Y]$ denote the set of $G$-homotopy classes of $G$-maps $X \rightarrow Y$. As usual, when $X$ is a (double) suspension, $[X, Y]$ has a natural (abelian) group structure. 
THEOREM G. Let $K$ and $L$ be connected $G$-spaces with $K$ a finite-dimensional $G$-complex and $\underline{\pi}_{1} L$ abelian. Then, there is a convergent spectral sequence $\left\{E_{p, q}^{r}\right\}$ such that

(a)

$$
\begin{aligned}
E_{p, q}^{2} & =\tilde{H}_{G}^{q}\left(K ; \underline{\pi}_{p+q} L\right), & & p \geq 1, q \geq 1, \\
& \subseteq \tilde{H}_{G}^{q}\left(K ; \underline{\pi}_{q} L\right), & & p=0, q \geq 1, \\
& =0, & & \text { otherwise, }
\end{aligned}
$$

where $\tilde{H}_{G}^{*}$ denotes reduced Bredon cohomology (see $[\mathbf{B}]$ or $\S 2$ ).

(b) For each $p \geq 1, E_{p, q}^{\infty}$ is the graded abelian group associated to a finite filtration of $\left[\sum^{p} K, L\right]$.

REMARKS. (1) Bredon [B, p. I-24] has a spectral sequence converging to $\tilde{H}_{G}^{q}\left(K ; \underline{\pi}_{p+q} L\right)$. When $K$ is a Moore $G$-space of type $(\underline{M}, n)$, this spectral sequence collapses, and we get

$$
\tilde{H}_{G}^{q}\left(K ; \underline{\pi}_{p+q} L\right)=\operatorname{Ext}^{q-n}\left(\underline{M} ; \underline{\pi}_{p+q} L\right)
$$

where $\mathrm{Ext}^{*}$ is a certain derived functor (see $\S 2$ ).

(2) Uniqueness is not usually a feature of homology decompositions, even in the nonequivariant case. Although it is possible, using the spectral sequence, to obtain conditions under which $k^{\prime}$-invariants are unique (up to $G$-homotopy), these conditions are too restrictive to admit a useful general formulation.

It is worth noting, however, that under the conditions of Theorem A, the Moore $G$-spaces that actually appear in a homology decomposition are unique up to $G$ equivalence. This follows from Theorem A of [K2]. We give some details in $\S 4$ after our proof of Theorem A.

The paper is organized as follows. In $\S 2$ we introduce terminology and derive the spectral sequence of Theorem G. In $\S 3$ we recall the construction of a homology decomposition, adapted to our equivariant context, and derive sufficient conditions for existence in terms of the spectral sequence. In $\S 4$ we prove all the results except Corollary B (which is evident) and Theorem F. The example in Theorem F is constructed in $\S 5$.

\section{An equivariant Federer spectral sequence.}

2.1. Terminology. Most of the terminology we present is explained in more detail in $[\mathbf{B}]$.

Let $O_{G}$ denote the category of canonical orbits of $G$, that is, the category whose objects are the left-coset spaces $G / H, H \leq G$, and whose morphisms are their $G$ maps. An $O_{G}$-object in a category $C$ is defined to be a contravariant functor from $O_{G}$ to $C$. When $C$ is a familiar category, we may modify this terminology accordingly. For example, when $C$ is the category Sets of sets and functions, or Top. of pointed spaces and pointed maps, or Abel of abelian groups and homomorphisms, we speak of $O_{G}$-sets, $O_{G}$-spaces, or $O_{G}$-modules, respectively. The category of all $O_{G}$-objects in $C$ and their natural transformations will be denoted $O_{G}^{\circ}(C)$.

If $C$ is the category Sets or Top. (or any one of a number of other related categories), we may consider the category $G-C$ of corresponding $G$-objects and $G$-maps. There is then defined a fixed-point functor $\Phi: G-C \rightarrow O_{G}^{\circ}(C)$ satisfying $\Phi(X)(G / H)=X^{H}, \Phi(f: X \rightarrow Y)(G / H)=f^{H}: X^{H} \rightarrow Y^{H}$. We may combine $\Phi$ with various standard functors in the following way. 
Suppose $F: C \rightarrow D$ is a (covariant) functor, and use the same name for the functor $O_{G}^{\circ}(C) \rightarrow O_{G}^{\circ}(D)$ induced by composition with $F$. We then obtain a composite

$$
G-C \stackrel{\Phi}{\rightarrow} O_{G}^{\circ}(C) \stackrel{F}{\rightarrow} O_{G}^{\circ}(D),
$$

which we denote by $\underline{F}$. For example, when $C$ is $T o p$. and $F$ is the reduced (singular) homology functor $\tilde{H}_{n}:$ Top. $\rightarrow$ Abel, then $\underline{\tilde{H}}_{n}$ is the homology functor described in the introduction. Similarly if $F$ equals $\pi_{n}$.

For other examples, $C$ may consist of pointed $\mathrm{CW}$ complexes and cellular maps and $F$ may be the functor taking each $\mathrm{CW}$ complex to its $n$-skeleton, or $F$ may be the cellular chain complex functor $\underline{C}_{*}$. Finally, if $\mathcal{C}$ equals $\operatorname{Sets}, F:$ Sets $\rightarrow$ Abel may denote the free-abelian-group functor (which, in fact, we denote by $F$ ). Then we call $\underline{F}[X]$ the free $O_{G}$-module on the $G$-set $X$ (cf. $[\mathbf{B}, \mathrm{p}$. I-23]). If $X$ indexes the $n$-cells of a $G$-complex $K$, then there is an isomorphism of $O_{G}$-modules $\underline{C}_{n}(K) \approx \underline{F}[X]$. Bredon shows that $\underline{F}[X]$ is projective as an $O_{G}$-module.

2.2. Setting up the spectral sequence. This construction follows closely the usual nonequivariant case $[\mathbf{F}]$ with some technical differences in the computations. Since we are particularly interested in the sequence for small values of $p$, where the construction is a bit delicate, we shall go into some detail.

Without loss of generality, we may assume that $K$ has precisely one 0-cell (cf. [B, Proposition 7.1]). Now proceed just as in the nonequivariant case and filter $K$ by skeleta

$$
*=K^{0} \subset K^{1} \subset \cdots \subset K^{q-1} \subset K^{q} \subset \cdots \subset K^{d}=K,
$$

where each $K^{q}$ is obtained from $K^{q-1}$ by equivariantly attaching a bouquet $D^{q} \wedge X_{q}^{+}$ of $q$-cells, $q \geq 1$. Here $X_{q}$ is a $G$-set and $X_{q}^{+}$is the pointed $G$-set obtained from it by adjoining a disjoint ( $G$-fixed) basepoint.

For each $q \geq 1$ we obtain an exact equivariant Puppe sequence, as in $[\mathbf{B}, \mathrm{p}$. III.4],

$$
\begin{aligned}
{\left[S^{q-1} \wedge X_{q}^{+}, L\right] } & \stackrel{f}{\leftarrow}\left[K^{q-1}, L\right] \stackrel{e}{\leftarrow}\left[K^{q}, L\right] \stackrel{g}{\leftarrow}\left[S^{q} \wedge X_{q}^{+}, L\right] \stackrel{f}{\leftarrow} \\
& \ldots \stackrel{g}{\leftarrow}\left[S^{p+q-1} \wedge X_{q}^{+}, L\right] \stackrel{f}{\leftarrow}\left[\sum^{p} K^{q-1}, L\right] \stackrel{e}{\leftarrow}\left[\sum^{p} K^{q}, L\right] \\
& \stackrel{g}{\leftarrow}\left[S^{p+q} \wedge X_{q}^{+}, L\right] \stackrel{f}{\leftarrow} \cdots .
\end{aligned}
$$

Define $A=\bigoplus_{p, q} A_{p, q}, E^{1}=\bigoplus_{p, q} E_{p, q}^{1}, p, q \in \mathbf{Z}$, as follows.

$$
\begin{aligned}
& A_{p, q}= \begin{cases}{\left[\sum^{p} K^{q}, L\right],} & p \geq 1, q \geq 1, \\
0, & \text { otherwise. }\end{cases} \\
& E_{p, q}^{1}= \begin{cases}{\left[S^{p+q} \wedge X_{q}^{+}, L\right],} & p \geq 1, q \geq 1, \\
\operatorname{ker} g \subset\left[S^{q} \wedge X_{q}^{+}, L\right], & p=0, q \geq 1, \\
0, & \text { otherwise. }\end{cases}
\end{aligned}
$$

Note that all the $A_{p, q}$ 's are groups and the $E_{p, q}^{1}$ 's abelian groups. The Puppe sequences splice together in the usual way to give an exact couple

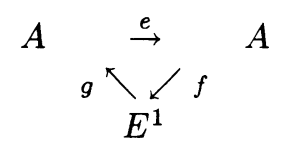


just as in Federer $[\mathbf{F}]$. The bidegrees of $e, f$, and $g$ are $(0,-1),(-1,1)$, and $(0,0)$, respectively. The spectral sequence is now obtained as in $[\mathbf{F}]$, converging to the graded group associated to a filtration $G_{p, q}=\operatorname{ker}\left(\left[\sum^{p} K, L\right] \rightarrow A_{p, q}\right), p \geq 1$, i.e.,

$$
\left[\sum^{p} K, L\right]=G_{p,-1} \supset G_{p, 0} \supset G_{p, 1} \supset \cdots, \quad p \geq 1,
$$

with $G_{p, q-1} / G_{p, q}=E_{p, q}^{\infty}$. The bidegree of $d^{r}$ is $(-1, r)$.

It remains for us to evaluate the $E^{2}$ term.

2.3. LEMMA.

$$
\begin{aligned}
E_{p, q}^{1} & =\tilde{C}_{G}^{q}\left(K ; \underline{\pi}_{p+q} L\right), & & p \geq 1, q \geq 0 \\
& \subseteq \tilde{C}_{G}^{q}\left(K ; \underline{\pi}_{q} L\right), & & p=0, q \geq 0 \\
& =0, & & \text { otherwise. }
\end{aligned}
$$

Here $\tilde{C}_{G}^{*}(K ; \underline{M})$ is defined as follows for any $O_{G}$-module $\underline{M}$. If $\underline{N}$ is another $O_{G}$-module, let $\operatorname{Hom}(\underline{N}, \underline{M})$ denote the abelian group of natural transformations $\underline{N} \rightarrow \underline{M}$. The $i$ th derived functor of Hom will be denoted $\operatorname{Ext}^{i}$. If $\underline{\tilde{C}}_{*} K=\underline{C}_{*}(K, *)$ denotes the reduced, cellular $O_{G}$-chain complex of $K$, then $\tilde{C}_{G}^{*}(K ; \underline{M})$ is the cochain complex $\operatorname{Hom}\left(\underline{\tilde{C}}_{*} K, \underline{M}\right)$.

PROOF OF 2.3. For $p \geq 1, q \geq 1$,

$$
E_{p, q}^{1}=\left[S^{p+q} \wedge X_{q}^{+}, L\right]=\operatorname{Hom}\left(\underline{F}\left[X_{q}\right], \underline{\pi}_{p+q} L\right),
$$

by Lemma 6.5 , p. II.15 of [B]. But, by definition, this last is $\tilde{C}_{G}^{q}\left(K ; \underline{\pi}_{p+q} L\right)$. The other cases are similar or easier.

2.4. LEMMA. $d^{1}=f \circ g: E_{p, q}^{1} \rightarrow E_{p-1, q+1}^{1}$ is induced, up to sign, by the standard coboundary map $\delta: \tilde{C}_{G}^{q}\left(K ; \underline{\pi}_{p+q} L\right) \rightarrow \tilde{C}_{G}^{q+1}\left(K ; \underline{\pi}_{p+q} L\right)$, for each $p \geq 0$, $q \geq 0$.

Here we are identifying $E_{p, q}^{1}$ with a subgroup of $\tilde{C}_{G}^{q}\left(K ; \underline{\pi}_{p+q} L\right)$ via Lemma 2.3.

PROOF. Note that for $q=0$ the result is trivially true, so assume $q \geq 1$. We first assume also that $p \geq 1$.

The composite (coming from the Puppe construction)

$$
S^{p+q} \wedge X_{q+1}^{+}=\sum^{p-1}\left(K^{q+1} / K q\right) \rightarrow \sum^{p} K^{q} \rightarrow \sum^{p}\left(K^{q} / K^{q-1}\right)=S^{p+q} \wedge X_{q}^{+}
$$

induces a commutative diagram

$$
\begin{array}{ccc}
{\left[S^{p+q} \wedge X_{q}^{+}, L\right]} & \stackrel{f \circ g}{\longrightarrow} & {\left[S^{p+q} \wedge X_{q+1}^{+}, L\right]} \\
\downarrow & & \\
\operatorname{Hom}\left(\underline{\pi}_{p+q} S^{p+q} \wedge X_{q}^{+}, \underline{\pi}_{p+q} L\right) & \rightarrow & \operatorname{Hom}\left(\underline{\pi}_{p+q} S^{p+q} \wedge X_{q+1}^{+}, \underline{\pi}_{p+q} \underline{L}\right)
\end{array}
$$

The bottom horizontal arrow of (2) is equivalent to the map

$$
\operatorname{Hom}\left(\underline{\tilde{H}}_{p+q}\left(S^{p+q} \wedge X_{q}^{+}\right), \underline{\pi}_{p+q} L\right) \rightarrow \operatorname{Hom}\left(\underline{\tilde{H}}_{p+q}\left(S^{p+q} \wedge X_{q+1}^{+}\right), \underline{\pi}_{p+q} L\right)
$$

which is obtained by applying the functor $\operatorname{Hom}\left(, \underline{\pi}_{p+q} L\right)$ to the composite

$$
\underline{\tilde{H}}_{p+q}\left(S^{p+q} \wedge X_{q+1}^{+}\right) \rightarrow \underline{\tilde{H}}_{p+q}\left(\sum \sum^{p} K^{q}\right) \rightarrow \underline{\tilde{H}}_{p+q}\left(S^{p+q} \wedge X_{q}^{+}\right)
$$


induced by (1). But the composite (3) is, up to sign and after desuspending, the cellular boundary map $\underline{\tilde{C}}_{q+1}(K) \rightarrow \underline{\tilde{C}}_{q}(K)$. Thus, the bottom row of (2) equals

$$
\tilde{C}_{G}^{q}\left(K ; \underline{\pi}_{p+q} L\right) \stackrel{ \pm \delta}{\rightarrow} \tilde{C}_{G}^{q+1}\left(K ; \underline{\pi}_{p+q} L\right) .
$$

The vertical arrows at either end of (2) are bijective, by Lemma 6.5, p. II.15 of [B], as in the proof of Lemma 2.3.

When $p=0$, we modify the above argument as follows. First, in (1),

$$
\sum^{p-1}\left(K^{q+1} / K^{q}\right)
$$

no longer makes sense, but $S^{p+q} \wedge X_{q+1}^{+}$does. The map $S^{p+q} \wedge X_{q+1}^{+} \rightarrow \sum^{p} K^{q}$ is simply the attaching map for the $(q+1)$-cells of $K$. For $q \geq 2$ proceed exactly as before. When $p=0$ and $q=1$, modify the bottom horizontal arrow of (2) by replacing $\underline{\pi}_{p+q}$ by $\underline{\tilde{H}}_{p+q}$ throughout the row. The argument then continues just as before, implying that the bottom arrow of (the modified) (2) equals

$$
\tilde{C}_{G}^{q}\left(K ; \underline{\tilde{H}}_{p+q} L\right) \stackrel{ \pm \delta}{\longrightarrow} \tilde{C}_{G}^{q+1}\left(K ; \underline{\tilde{H}}_{p+q} L\right), \quad p=0, q=1 .
$$

The assumption that $\underline{\pi}_{1} L$ is abelian now allows us to replace $\underline{\tilde{H}}_{p+q} L$ by $\underline{\pi}_{p+q} L$, so our conclusion is the same as before, with the exception that the vertical arrows at either end of (2) are now known only to be injective, as in 2.3.

2.5. COROLlaRY.

$$
\begin{aligned}
E_{p, q}^{2} & =\tilde{H}_{G}^{q}\left(K ; \underline{\pi}_{p+q} L\right), & & p \geq 1, q \geq 0 \\
& \subseteq \tilde{H}_{G}^{q}\left(K ; \underline{\pi}_{q} L\right), & & p=0, q \geq 0 \\
& =0, & & \text { otherwise. }
\end{aligned}
$$

Here $\tilde{H}_{G}^{*}$ denotes (reduced) Bredon cohomology, which equals the homology of the cochain complex $\tilde{C}_{G}^{*}$.

ProOF. Except for the case $p=0$, the result is immediate from 2.3 and 2.4. When $p=0$, we look at the commutative diagram

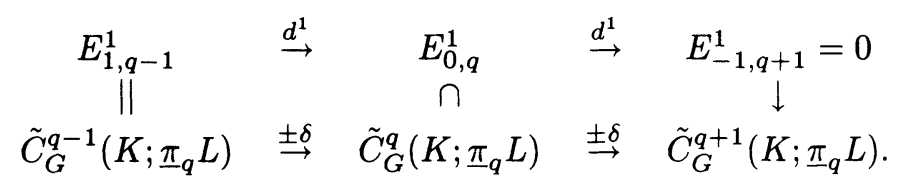

Clearly, $E_{0, q}^{2}=E_{0, q}^{1} / d^{1}\left(E_{1, q-1}^{1}\right) \subset \operatorname{ker} \delta / \operatorname{im} \delta=\tilde{H}_{G}^{q}\left(K ; \underline{\pi}_{q} L\right)$. G.

This completes our discussion of the spectral sequence and our proof of Theorem

3. Equivariant homology decomposition. Here we outline the construction of an equivariant homology decomposition of $X$, in preparation for the proof of Theorem A. Suppose that conn $X=c-1 \geq 1$.

For $m \leq c-1$, set $K_{m}=*$. It is easy to see that a homology decomposition must begin with a Moore $G$-space $K_{c}$ which is a suspension and is of type $\left(\underline{\tilde{H}}_{c} X, c\right)$. We suppose this exists and attempt to find a $G$-map

$$
K_{c} \stackrel{e_{c}}{\rightarrow} X
$$

inducing an $\underline{\tilde{H}}_{c}$-equivalence. 
Next, assuming that we have constructed a partial homology decomposition

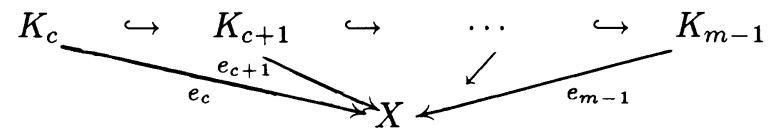

we try to find a Moore $G$-space $A_{m}$ of type $\left(\underline{\tilde{H}}_{m} X, m-1\right)$ and to form a pushout square

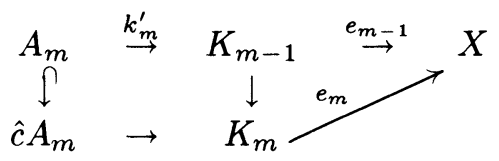

in such a way that $e_{m-1}$ extends to a $G$-map $e_{m}: K_{m} \rightarrow X$ with the required properties.

As in the nonequivariant case, one sees that such a pushout can be constructed if and only if the solid arrow diagram

$$
\begin{array}{ccc}
A_{m} & \stackrel{k_{m}^{\prime}}{\rightarrow} & K_{m-1} \\
\downarrow & & \downarrow e_{m-1} \\
\hat{c} A_{m}- & k_{m}^{\prime \prime}-\rightarrow & X
\end{array}
$$

can be completed so as to induce an equivalence

$$
\underline{H}_{m}\left(\hat{c} A_{m}, A_{m}\right) \approx \underline{H}_{m}\left(X, K_{m-1}\right) \quad\left(\approx \underline{\tilde{H}}_{m} X\right) .
$$

Now let $\Phi\left(e_{m-1}\right)$ denote the homotopy-fibre of $e_{m-1}$, i.e.,

$$
\Phi\left(e_{m-1}\right)=K_{m-1} \times_{X} P(X),
$$

where $P(X)$ is the pointed $G$-space of based paths in $X$. Clearly, $\Phi\left(e_{m-1}\right)$ inherits the structure of a pointed $G$-space, and there is a bijective correspondence between diagrams (3.4) and pointed $G$-maps

$$
A_{m} \stackrel{k_{m}^{*}}{\rightarrow} \Phi\left(e_{m-1}\right) .
$$

The correspondence respects $G$-homotopy, and (3.4) induces an $\underline{H}_{m}$-equivalence if and only if (3.5) induces an $\underline{\tilde{H}}_{m-1}$-equivalence. Note that both $\underline{\tilde{H}}_{m-1} A_{m}$ $\left(=\underline{\pi}_{m-1} A_{m}\right)$ and $\underline{\tilde{H}}_{m-1} \Phi\left(e_{m-1}\right)\left(=\underline{\pi}_{m-1} \Phi\left(e_{m-1}\right)\right)$ are isomorphic to $\underline{\tilde{H}}_{m} X$.

Thus, we look for conditions which imply that the canonical map

$$
\left[A_{m}, \Phi\left(e_{m-1}\right)\right] \rightarrow \operatorname{Hom}\left(\underline{\tilde{H}}_{m-1} A_{m}, \underline{H}_{m-1} \Phi\left(e_{m-1}\right)\right)
$$

maps onto an equivalence. It is, of course, sufficient for the map to be surjective.

Assume that $A_{m}$ is a suspension $\sum B_{m}$, and replace $\underline{\tilde{H}}_{m-1} \Phi\left(e_{m-1}\right)$ by $\underline{\pi}_{m-1} \Phi\left(e_{m-1}\right)$. Then, (3.6) becomes

$$
\left[\sum B_{m}, \Phi\left(e_{m-1}\right)\right] \rightarrow \operatorname{Hom}\left(\underline{\tilde{H}}_{m-2} B_{m}, \underline{\pi}_{m-1} \Phi\left(e_{m-1}\right)\right),
$$

which comes from the Federer spectral sequence of $\S 2$ in the following way: $\left[\sum B_{m}, \Phi\left(e_{m-1}\right)\right]$ is filtered

$$
\left[\sum B_{m}, \Phi\left(e_{m-1}\right)\right]=G_{1,-1} \supset G_{1,0} \supset G_{1,1} \supset \cdots,
$$


with $G_{1,-1}=G_{1,0}=\cdots=G_{1, m-3} \supset G_{1, m-2}$, by the connectivity of $B_{m}$, and

$$
G_{1, m-3} / G_{1, m-2}=E_{1, m-2}^{\infty} \subset E_{1, m-2}^{2}=\tilde{H}_{G}^{m-2}\left(B_{m} ; \underline{\pi}_{m-1} \Phi\left(e_{m-1}\right)\right) .
$$

By Remark (1) following Theorem $G$ in $\S 1$, this last group is

$$
\operatorname{Hom}\left(\underline{\tilde{H}}_{m-2} B_{m}, \underline{\pi}_{m-1} \Phi\left(e_{m-1}\right)\right),
$$

and (3.7) may be identified with the composite

$$
\left[\sum B_{m}, \Phi\left(e_{m-1}\right)\right]=G_{1, m-3} \rightarrow G_{1, m-3} / G_{1, m-2}=E_{1, m-2}^{\infty} \hookrightarrow E_{1, m-2}^{2} .
$$

Therefore, we can find the desired equivalence (3.5) provided that the differentials

$$
d^{r}: E_{1, m-2}^{r} \rightarrow E_{0, m+r-2}^{r}
$$

vanish, for all $r \geq 2$. Now $E_{0, m+r-2}^{r}$ is a quotient of $E_{0, m+r-2}^{2}$, which, by Corollary 2.5 , is contained in

$$
\tilde{H}_{G}^{m+r-2}\left(B_{m} ; \underline{\pi}_{m+r-2} \Phi\left(e_{m-1}\right)\right)=\operatorname{Ext}^{r}\left(\underline{\tilde{H}}_{m-2} B_{m}, \underline{\pi}_{m+r-2} \Phi\left(e_{m-1}\right)\right) .
$$

Thus, a sufficient condition for the existence of the desired map $k_{m}^{*}$ is that

$$
\operatorname{Ext}^{r}\left(\underline{\tilde{H}}_{m-2} B_{m}, \underline{\pi}_{m+r-2} \Phi\left(e_{m-1}\right)\right)
$$

be 0 . Note that $\underline{\tilde{H}}_{m-2} B_{m}=\underline{\tilde{H}}_{m-1} A_{m}=\underline{\tilde{H}}_{m} X$.

Completely analogous considerations apply to the problem of finding the $\underline{H}_{c^{-}}$ equivalence (3.1). Only now $A_{c}=K_{c}$ is a Moore space of type $\left(\underline{\tilde{H}}_{c} X, c\right)$ (not $\left.\left(\underline{\tilde{H}}_{c} X, c-1\right)\right)$ and $\Phi$ is replaced by $X$. In this case, the sufficient condition is that $\operatorname{Ext}^{r}\left(\underline{\tilde{H}}_{c} X, \underline{\pi}_{c+r-1} X\right)$ be 0 .

3.10. Summary. Suppose that $X$ is a pointed $G$-space with $\underline{\operatorname{conn}} X=c-1 \geq 1$. The following conditions are sufficient for the existence of a homology decomposition of $X$.

(a) Moore $G$-spaces of types $\left(\underline{\tilde{H}}_{c} X, c\right)$ and $\left(\underline{\tilde{H}}_{m} X, m-1\right)$, for all $m>c$, exist and are suspensions.

(b) $\operatorname{Ext}^{r}\left(\underline{\tilde{H}}_{c} X, \underline{\pi}_{c+r-1} X\right)=0$, for all $r \geq 2$.

(c) For each $m>c$, a partial homology decomposition (3.2) exists and

$$
\operatorname{Ext}^{r}\left(\underline{\tilde{H}}_{m} X, \underline{\pi}_{m+r-2} \Phi\left(e_{m-1}\right)\right)=0,
$$

for all $r \geq 2$.

Theorem A simply replaces (a)-(c) with more readily verifiable conditions. We next give the proof.

4. Proofs of A, C, D, E.

ProOF OF THEOREM D. Recall that, by hypothesis, $X$ is a pointed $G$-space with $\underline{\text { conn }} X \geq 1$ and proj $\operatorname{dim} \underline{\tilde{H}}_{m} X \leq 1$ for each $m$. Therefore, by 3.10 , it suffices to verify that Moore $G$-spaces of all types $(\underline{M}, n), n \geq 2$, exist and are suspensions, provided $\operatorname{proj} \operatorname{dim} \underline{M} \leq 1$. This is proved by an equivariant version of a standard, direct cell-attachment argument (Theorem $4.2(\mathrm{a})$ and $\S 5$ of $[\mathbf{K 2}]$ ), using a short, free resolution of $\underline{M}$ to prescribe the attaching map. (Although the theorems in $[$ K2] are proved subject to condition $(\mathbf{Q})$, this plays no role in the proof of $4.2(\mathrm{a})$, nor in the relevant construction in $\S 5$.)

The proof of Theorem A requires the following well-known fact. 
LEMMA. Let $L$ be an $n$-connected rational $C W$ complex, $n \geq 1$, and suppose that $i \leq 2 n$. Then the Hurewicz homomorphism $\pi_{i} L \rightarrow \tilde{H}_{i} L$ is bijective.

PROOF OF THEOREM A. We proceed to verify the conditions of 3.10 under the given hypotheses.

Condition (a). We begin with the case conn $X=c-1=1$. The hypotheses of the theorem then force proj $\operatorname{dim} \underline{\tilde{H}}_{m} X \leq 1$, , for all $m$, and we have already shown above that all required Moore $G$-spaces exist and are suspensions in this case.

When conn $X=c-1>1$, we need only find appropriate Moore $G$-spaces of type $(\underline{M}, n)$ for $n \geq 3$. Under condition (Q), a theorem of Triantafillou [T1] implies the existence of Moore $G$-spaces of type $(\underline{M}, 2)$, and we suspend these sufficiently often.

Conditions (b) and (c). We begin by evaluating $\underline{\pi}_{m+r-2} \Phi\left(e_{m-1}\right)$, assuming $1 \leq r \leq$ conn $X \leq m-1$. Of course, by construction,

$$
\pi_{m+r-2} \Phi\left(e_{m-1}\right)=\underline{\pi}_{m+r-1}\left(X, K_{m-1}\right) .
$$

Since $r \leq \underline{\operatorname{conn}} X \leq m-1$, we have $\underline{\text { conn }} K_{m-1}=\underline{\text { conn }} X$, and the Blakers-Massey Theorem applies:

$$
\underline{\pi}_{m+r-1}\left(X, K_{m-1}\right)=\underline{\pi}_{m+r-1}\left(X / K_{m-1}\right) .
$$

Now $\underline{\operatorname{conn}}\left(X / K_{m-1}\right) \geq m-1$, so the above lemma gives

$$
\begin{aligned}
\underline{\pi}_{m+r-1}\left(X / K_{m-1}\right) & =\underline{\tilde{H}}_{m+r-1}\left(X / K_{m-1}\right) \\
& =\underline{H}_{m+r-1}\left(X, K_{m-1}\right)=\underline{\tilde{H}}_{m+r-1} X,
\end{aligned}
$$

for all $r \leq \underline{\text { conn }} X$. Thus, $\underline{\pi}_{m+r-2} \Phi\left(e_{m+1}\right)=\underline{\tilde{H}}_{m+r-1} X$.

The lemma also implies that $\underline{\pi}_{c+r-1} X=\underline{\tilde{H}}_{c+r-1} X$ when $r \leq \underline{\text { conn }} X=c-1$.

We now verify the algebraic conditions in $3.10(\mathrm{~b})$, (c). An inductive construction as in $\S 3$ then constructs the decomposition. Consider

(1) $\operatorname{Ext}^{r}\left(\underline{\tilde{H}}_{c} X, \underline{\pi}_{c+r-1} X\right), c-1=\underline{\operatorname{conn}} X$, and

(2) $\operatorname{Ext}^{r}\left(\underline{\tilde{H}}_{m} X, \underline{\pi}_{m+r-2} \Phi\left(e_{m-1}\right)\right), m>c$.

Of course, if $r>\operatorname{proj} \operatorname{dim} \underline{\tilde{H}}_{i} X, i=c$ or $m$, then (1) or (2) is zero, respectively, so assume $r \leq \operatorname{proj} \operatorname{dim} \underline{\tilde{H}}_{i} X, i=c$ or $m$. In either case we get $r \leq \underline{\operatorname{conn}} X$ so that both (1) and (2) reduce to

(3) $\operatorname{Ext}^{r}\left(\underline{\tilde{H}}_{i} X, \underline{\tilde{H}}_{i+r-1} X\right)$

by the above computation. But, we also have $\operatorname{proj} \operatorname{dim} \underline{\tilde{H}}_{i} X \leq \gamma(i, X)$, so that $\tilde{H}_{i+r-1} X=0$, for every $r$ satisfying $2 \leq r \leq \operatorname{proj} \operatorname{dim} \underline{\tilde{H}}_{i} X$. Thus (3) is zero and our verification is complete.

Remark on uniqueness of Moore $G$-spaces. Suppose that conn $X=c-1 \geq 1$ and set

$$
\left(\underline{M}_{m}, n_{m}\right)= \begin{cases}\left(\underline{\tilde{H}}_{m} X, m\right), & m \leq c, \\ \left(\underline{\tilde{H}}_{m} X, m-1\right), & m>c .\end{cases}
$$

The only Moore $G$-spaces appearing in a homology decomposition of $X$ are of types $\left(\underline{M}_{m}, n_{m}\right), m \geq 2$. The hypotheses of Theorem A imply that

$$
\operatorname{proj} \operatorname{dim} \underline{M}_{m}+1 \leq n_{m}, \quad m \geq 2,
$$


and this is precisely the hypothesis for the uniqueness theorem (Theorem A of [K2]). Thus, under the hypotheses of Theorem A, the said Moore $G$-spaces are unique up to $G$-equivalence.

Note that, in contrast to the nonequivariant case, uniqueness does not hold in general for Moore $G$-spaces (Theorem B of $[\mathbf{K 2}]$ ).

PROOF OF COROLlaRY C. (a) When $G$ is a cyclic $p$-group, every rational $O_{G^{-}}$ module has projective dimension $\leq 1$ [T3, T2]. The result now follows immediately from Theorem A.

(b) When $G=\mathbf{Z} / p+\mathbf{Z} / p$, every rational $O_{G}$-module has projective dimension $\leq 2$ [T3, T2]. Again, the result is an immediate consequence of Theorem A.

(c) For any finite $G$, let $l(G)$ denote the largest $l$ for which there exists a chain of subgroups

$$
e=G_{0}<G_{1}<\cdots<G_{l}=G .
$$

Since $\left|G_{i}: G_{i+1}\right| \geq 2, l(G) \leq \log _{2}|G|$. A lemma of $[\mathbf{R} \& \mathbf{T}]$ shows that, assuming (Q), $l(G)$ is an upper bound for the projective dimension of $\mathcal{O}_{G}$-modules.

If $K$ is a rational Eilenberg-Mac Lane $G$-space of type $(\underline{\pi}, n)$, then it is well known that $\underline{\tilde{H}}_{i} K=0$ except when $i \equiv 0(\bmod n)$. Thus, either $\underline{H}_{m} K=0$ or $\gamma(m, K)=n$. The assumption $n>\log _{2}|G|$ (or, better, $n>l(G)$ ) then insures that proj $\operatorname{dim} \underline{\tilde{H}}_{m} K \leq \min \{\underline{\text { conn }} K, \gamma(m, K)\}$, for all $m$, so that Theorem A applies.

PROOF OF COROLlaRY E. Let $X$ be given as in the corollary. Then there exists a pointed $G$-space $Y$ and a $G$-map $f: Y \rightarrow X$ such that

(1) $Y^{H}=*$, unless $H=e$;

(2) $f$ is a weak homotopy equivalence of spaces.

In fact, $Y$ is obtained from the product $X \times E, E$ a contractible, free (unpointed) $G$-space, by adjoining the cone on $* \times E$. The map $f$ is the trivial extension of the projection $X \times E \rightarrow X$. This construction is also used in [K1 and K2].

Note that $Y$ satisfies

(3) $\underline{\tilde{H}}_{m} Y(G / H)=0$, unless $H=e$;

(4) $\underline{\tilde{H}}_{m} Y(G / e)=\tilde{H}_{m} X$.

Using hypothesis (b) of the corollary, together with a theorem of Rim [Ri], this last has proj $\operatorname{dim} \leq 1$, as a $\mathbf{Z} G$-module. Let

$$
0 \rightarrow P_{1} \stackrel{\partial_{1}}{\rightarrow} P_{0} \stackrel{\partial_{0}}{\rightarrow} \tilde{H}_{m} X \rightarrow 0
$$

be a length-one projective $\mathbf{Z} G$-resolution.

We define a length-one projective $O_{G}$-resolution of $\underline{\tilde{H}}_{m} Y$

$$
0 \rightarrow \underline{P}_{1} \stackrel{\underline{\partial}_{1}}{\rightarrow} \underline{P}_{0} \stackrel{\underline{\partial}_{0}}{\rightarrow} \underline{\tilde{H}}_{m} Y \rightarrow 0
$$

as follows. Let

$$
\underline{P}_{i}(G / H)= \begin{cases}0, & \text { unless } H=e, \quad i=0,1, \\ P_{i}, & H=e,\end{cases}
$$

with (the only possible) trivial maps $\underline{P}_{i}(G / K) \rightarrow \underline{P}_{i}(G / H)$ corresponding to morphisms $G / H \rightarrow G / K, H<K$. It is not hard to check that these $O_{G}$-modules are projective. The maps $\underline{\partial}_{i}$ of $(6), i=0,1$, are given at $G / e$ by the $\partial_{i}$ in (5).

Thus, proj $\operatorname{dim} \underline{\tilde{H}}_{m} Y \leq 1$, for all $m$, and Theorem D applies to produce a homology decomposition $*=K_{1} \subset K_{2} \subset \cdots \subset K \stackrel{e}{\rightarrow} Y$. The composite $K \stackrel{f \circ e}{\rightarrow} X$ then gives the desired classical equivariant homology decomposition of $X$. 


\section{A $G$-space with no homology decomposition.}

5.1. Algebra. In this section we construct an $O_{G}$-chain complex which will be the cellular chain complex of our example.

Choose $G=\mathbf{Z} / 2 \oplus \mathbf{Z} / 2$. A typical $O_{G}$-module $\underline{M}$ may be represented as follows:

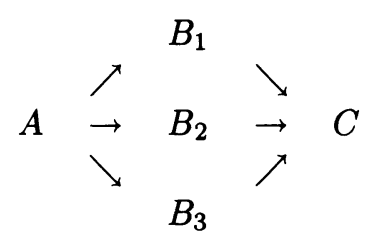

where $A=\underline{M}(G / G)$ is an abelian group, $C=\underline{M}(G / e)$ is a $\mathbf{Z}[G]$-module, and $B_{i}=\underline{M}\left(G / H_{i}\right)$ is a $\mathbf{Z}\left[G / H_{i}\right]$-module, $i=1,2,3$, with $H_{1}, H_{2}, H_{3}$ denoting the three order-two subgroups of $G$. The arrows in the diagram denote appropriate module homomorphisms. (See [K2 or T3] for more details.) When the maps are understood or unimportant, we abbreviate the above by

$$
\begin{array}{ccc} 
& B_{1} & \\
A & B_{2} & C \\
& B_{3} &
\end{array}
$$

We now assume condition $(\mathbf{Q})(\mathbf{1})$, so that all abelian groups become $\mathbf{Q}$-vector spaces, modules are $\mathbf{Q}[G]$ or $\mathbf{Q}[G / H]$-modules, etc. $\mathbf{Q}$ itself will be given the trivial $\mathbf{Q}[G / H]$ structure for all $H \leq G$. Set

$$
\underline{M}=\mathbf{Q} \begin{array}{ccc}
0 & \\
\mathbf{Q} & 0 \\
0
\end{array} \quad \text { and } \quad \underline{P}_{0}=\mathbf{Q} \begin{aligned}
& \mathbf{Q} \\
& \mathbf{Q}
\end{aligned} \quad \mathbf{Q}
$$

with all maps in $\underline{P}_{0}$ the identity. $\underline{P}_{0}$ is projective. In fact, $\underline{P}_{0}$ is the free, rational $O_{G}$-module on the singleton $G$-set $\{\sigma\}:$ if $\mathbf{Q}:$ Sets $\rightarrow \mathbf{Q}$-vector-spaces is the functor that sends the set $X$ to the $\mathbf{Q}$-vector-space $\mathbf{Q}[X]$ on the basis $X$, then we can write $\underline{P}_{0}=\underline{\mathbf{Q}}[\{\sigma\}]$, following the notation of 2.1.

We now construct a minimal projective resolution of $\underline{M}$ using the projective-cover construction of [T2]. To do this, we need to define two more projective $O_{G}$-modules. First, define

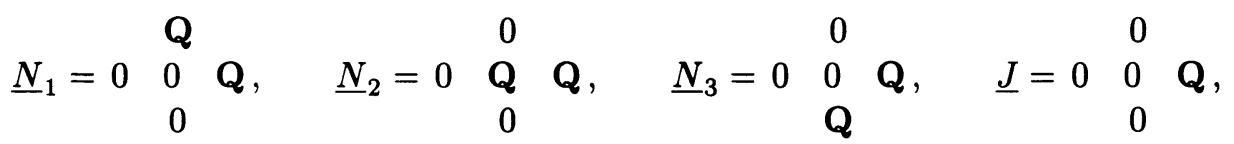

with all maps standard inclusions. All are projective. Set

$$
\underline{P}_{1}=\underline{N}_{1} \oplus \underline{N}_{2} \oplus \underline{N}_{3}=0 \begin{array}{ll}
\mathbf{Q} \\
\mathbf{Q}
\end{array} \mathbf{Q}^{3},
$$

and

$$
\underline{P}_{2}=\underline{J}^{2}=\underline{J} \oplus \underline{J}=0 \begin{array}{ll}
0 & 0 \\
& 0
\end{array}
$$


The resolution can now be written down as

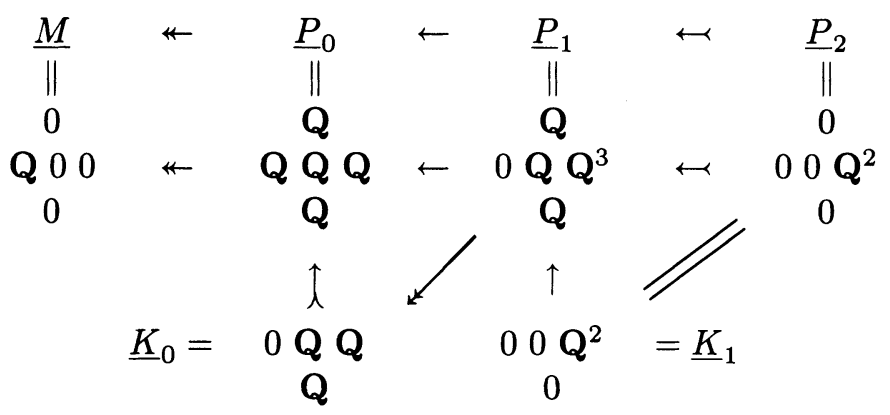

$\underline{P}_{0}$ is the projective cover of $\underline{M}, \underline{K}_{0}$ is the corresponding kernel. $\underline{P}_{1}$ is the projective cover of $\underline{K}_{0}$, etc. It follows that the resolution (1) is minimal.

Next, we add complementary modules to the $\underline{P}_{i}$ to make the resolution free.

$$
\underline{M} \hookleftarrow \underline{F}_{0} \stackrel{\underline{\partial}_{1}}{\rightarrow} \underline{F}_{1} \stackrel{\stackrel{\partial}{2}_{2}}{\mapsto} F_{2} .
$$

Write $\underline{F}_{i}=\underline{F}\left[X_{i}\right]$, for some $G$-sets $X_{i}, i=1,2,3$.

Consider the chain complexes

$$
\underline{C}: \underline{F}_{0} \stackrel{\underline{\partial}_{1}}{\leftarrow} \underline{F}_{1} \stackrel{\underline{\partial}_{2}}{\leftarrow} \underline{F}_{2}
$$

and

$$
\underline{D}: \underline{F}_{0} \stackrel{\underline{\partial}_{1}}{\leftarrow} \underline{F}_{1}
$$

obtained from (2). We shall realize both of these. The realization $L$ of $D$ will be our example.

5.2. LEMMA. Every chain map $\underline{\phi} \underline{C} \rightarrow \underline{D}$ induces the zero homomorphism $\underline{\phi}_{*}: H_{0} \underline{C} \rightarrow H_{0} \underline{D}$.

ProOF. Suppose the conclusion is false. Since by construction $H_{0} \underline{C}=\underline{M}=$ $H_{0} \underline{D}$, there exists a nonzero rational number $r$ such that $r \cdot \phi$ is a chain map inducing the identity $H_{0} \underline{C} \rightarrow H_{0} \underline{D}$. Thus, we may as well assume that $\underline{\phi}$ already has this property.

Let $\underline{i}: \underline{D} \rightarrow \underline{C}$ denote the inclusion. This is a chain map, hence, so is $\underline{i} \circ \phi$ : $\underline{C} \rightarrow \underline{C}$. Since $\underline{C}$ is a projective resolution of $\underline{M}$ and $\underline{i} \circ \phi$ induces the identity, $\underline{i} \circ \phi$ is chain-homotopic to $\mathrm{id}_{\underline{C}}$. In particular, there is a homomorphism $\underline{s}: \underline{F}_{1} \rightarrow \underline{F_{2}}$ such that $\operatorname{id}_{\underline{C}}-\underline{i} \circ \underline{\phi}=\underline{s}^{\circ} \underline{\partial}_{2}$ as maps $\underline{F}_{2} \rightarrow \underline{F}_{2}$. Since $\underline{i} \circ \underline{\phi}$ is 0 on $\underline{F}_{2}$, id $\underline{C}=\underline{s}_{0} \circ \underline{\partial}_{2}$, i.e., $\underline{F}_{2}$ is a direct summand of $\underline{F}_{1}$.

It follows that $\underline{M}$ has a projective resolution of length one, contradicting the fact that (1) was constructed as a minimal projective resolution.

5.3. Realizing the chain complexes. We use $\underline{\partial}_{1}$ and $\underline{\partial}_{2}$ to construct $G$-maps

$$
S^{2} \wedge X_{1}^{+} \stackrel{d_{1}}{\rightarrow} S^{2} \wedge X_{0}^{+}, \quad S^{3} \wedge X_{2}^{+} \stackrel{d_{2}}{\rightarrow} \hat{c}\left(d_{1}\right)
$$

where $\hat{c}\left(d_{1}\right)$ is the reduced mapping cone of $d_{1}$.

More precisely, note that there are canonical isomorphisms

$$
\begin{aligned}
& \underline{\pi}_{2}\left(S^{2} \wedge X_{0}^{+}\right)=\underline{F}_{0}=\underline{F}\left[X_{0}\right], \\
& \underline{\pi}_{2}\left(S^{2} \wedge X_{1}^{+}\right)=\underline{F}_{1}=\underline{F}\left[X_{1}\right], \\
& \underline{\pi}_{3}\left(S^{3} \wedge X_{2}^{+}\right)=\underline{F}_{2}=\underline{F}\left[X_{2}\right] .
\end{aligned}
$$


Thus, $\underline{\partial}_{1}$ may be regarded as a map of $\mathcal{O}_{G}$-modules $\underline{F}_{1} \rightarrow \underline{\pi}_{2}\left(S^{2} \wedge X_{0}^{+}\right)$, and this may be represented by a $G$-map $d_{1}$, unique up to $G$-homotopy, using Lemma 6.5 , p. II.15, of $[\mathbf{B}]$.

The mapping cone $\hat{c}\left(d_{1}\right)$ is 1 -connected and satisfies $\underline{\tilde{H}}_{3} \hat{c}\left(d_{1}\right)=\operatorname{ker} \underline{\partial}_{1}=\operatorname{im} \underline{\partial}_{2}$. Thus, we have a diagram

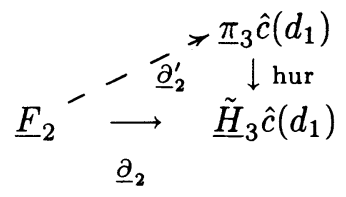

in which hur, the Hurewicz homomorphism, is an epimorphism of $O_{G}$-modules (i.e., $\operatorname{hur}(G / H)$ is surjective for each $H \leq G)$. Since $\underline{F}_{2}$ is projective, the diagram may be completed. Now apply the same result of $[\mathbf{B}]$ to $\underline{\partial}_{2}^{\prime}$, obtaining $d_{2}$.

Set $L=\sum \hat{c}\left(d_{1}\right)$ and $K=\sum \hat{c}\left(d_{2}\right)$. The (reduced) cellular chain complexes $\underline{\tilde{C}}_{*} K$ and $\underline{\tilde{C}}_{*} L$ are precisely $\underline{C}$ and $\underline{D}$, respectively, modulo a dimension shift of 3 . Note that $K$ is a Moore $G$-space of type $(\underline{M}, 3)$.

\subsection{Proposition. L does not admit a homology decomposition.}

PROOF. $L$ is 2-connected and $\underline{\tilde{H}}_{3} L=\underline{M}$. We show that there is no Moore $G$ space $K_{3}$ of type $(\underline{M}, 3)$ admitting a $G$-map $e_{3}: K_{3} \rightarrow L$ which is an $\underline{\tilde{H}}_{3}$-equivalence. Thus, a homology decomposition of $L$ cannot get started.

Since $\operatorname{proj} \operatorname{dim} \underline{M}=2$, Moore $G$-spaces of type $(\underline{M}, 3)$ are unique up to $G$ homotopy type $\left[\mathbf{K 2}\right.$, Theorem A]. Thus, we need only show that there is no $\underline{\tilde{H}}_{3}$ equivalence $e_{3}: K \rightarrow L$. But, if such an $e_{3}$ existed, it could be $G$-deformed into a cellular $G$-map. This would give a chain map $\underline{\tilde{C}}_{*} K \rightarrow \underline{\tilde{C}}_{*} L$ which induced an $\underline{\tilde{H}}_{3}$-equivalence, contradicting Lemma 5.2.

REMARK. The same argument applies to any suspension $\sum^{n} L$. In this case we need the uniqueness of Moore $G$-spaces of type $(\underline{M}, n+3)$, which also follows from Theorem A of [K2].

\section{REFERENCES}

[B] G. Bredon, Equivariant cohomology theories, Lecture Notes in Math., vol. 34, SpringerVerlag, New York, 1967.

[C] G. Carlsson, A counterexample to a conjecture of Steenrod, Invent. Math. 64 (1981), 171-174.

[F] H. Federer, A study of function spaces by spectral sequences, Amer. Math. Soc. Transl. 82 (1956), 340-361.

[H] P. J. Hilton, Homotopy theory and duality, Gordon \& Breach, New York, 1965.

[K1] P. J. Kahn, Steenrod's problem and k-invariants of certain classifying spaces, Algebraic $K$-theory, Part II, Lecture Notes in Math., vol. 967, Springer-Verlag, New York, 1982.

[K2] _ Rational Moore G-spaces, Trans. Amer. Math. Soc. 298 (1986), 245-271.

[M] J. Moore, Le théoreme de Freudenthal, la suite exacte de James, et l'invariante de Hopf généralise, Séminaire H. Cartan, Paris (1954-1955), Exposé 22 (Appendice) (10).

[Ri] D. S. Rim, Modules over finite groups, Ann. of Math. (2) 69 (1959), 700-712.

[Ro] C. Robinson, Moore-Postnikov systems for non-simple fibrations, Illinois J. Math. 16 (1972), 234-242.

[R \& T] M. Rothenberg and G. Triantafillou, An algebraic model for simple homotopy types, Univ. of Minnesota Math. Report, 1984, pp. 83-112. 
[Sc] R. Schultz, Homotopy decompositions of equivariant function spaces, Math. Z. 132 (1973), 69-90.

[Sm] J. Smith, Equivariant Moore spaces (preprint).

[T1] G. Triantafillou, G-spaces with prescribed equivariant cohomology, Univ. of Minnesota (preprint).

[T2] _ Equivariant minimal models, Trans. Amer. Math. Soc. 274 (1982), 509-532.

[T3] _ Rationalization of Hopf G-spaces, Math. Z. 182 (1983), 485-500.

Department of Mathematics, Cornell University, IthaCA, NeW York 14853 